\title{
A Proposal for Distributed Interactive Genetic Algorithm for Composition of Musical Melody
}

\author{
Makoto Fukumoto ${ }^{*}$, Takeshi Hatanaka ${ }^{\dagger}$
}

\begin{abstract}
This study proposes an Interactive Evolutionary Computation that creates sound contents for multiple users. Sound contents including music piece and sign sound are often used for creating common atmosphere and transmitting a certain message to everyone. The proposed method is based on parallel distributed Interactive Genetic Algorithm (IGA). As a special property of this method, in some generations, solution candidates are exchanged between the users. With the exchange, each of the users is affected by other users' feelings: good solutions for all of the users are expected to be obtained. Based on the proposed method, we constructed an IGA system for fundamentally investigating efficiencies of the proposed method. Aim of the IGA system is to create a short music melody commonly affording bright image to multiple users. Key of the notes was treated as gene of the IGA. Music chord progression is attached to the melody when it is presented to the subjects. In listening experiment, sixteen subjects divided into eight pairs, and two subjects participated in the evaluation process simultaneously. Experimental results showed increase in mean fitness values between the subjects.
\end{abstract}

Keywords: Interactive Genetic Algorithm, Multiple Users, Composition, Music Melody

\section{Introduction}

We use media contents in various situations in daily life. Using media contents suited to user's feeling will enrich our life by enhancing its effects. From this perspective, optimization of media contents is needed. Interactive Evolutionary Computation (IEC) is known as one of the methods [1][2]. IEC utilizes searching ability of Evolutionary Computation (EC), and the optimal solutions in EC are corresponded to media contents suited to the user in IEC. While most of IEC approaches in 1990s focused on senses of sight and hearing [1], recent IEC studies have been spreading its application areas to various senses of taste [3], smell [4][5][6][7], and touch [8][9][10].

IEC is originally used for optimizing media contents suited to each of the users' feelings. However, in some situations, media contents for multiple users are more important. Recent IEC studies proposed a method that creates media contents commonly suited to multiple users. These

\footnotetext{
* Fukuoka Institute of Technology, Fukuoka, Japan

${ }^{\dagger}$ Fukuoka Institute of Technology, Fukuoka, Japan
} 
previous IEC studies employed various schemes; summarizing multiple users' subjective feelings [11], gathering multiple users' votes [12][13][14], and employing island type evolutionary algorithm [15][16]. These previous studies employed Genetic Algorithm (GA) [17][18] as its evolutionary algorithm, thus, they are kind of Interactive Genetic Algorithm (IGA) [1]. Applications of these studies were limited in the area of images related to sense of sight.

This study aims to propose an IEC that creates sound contents for multiple users. Sound contents including music pieces and sign sounds are often used in our daily life for creating common atmosphere and transmitting a certain message to everyone. Target of the present study is to create music melodies suited to multiple users' feeling. The proposed method is based on parallel distributed IGA [15][16], which creates computer graphic contents commonly suited to multiple users. In the parallel distributed IGA, each of the users individually performs general IGA process by evaluating sound contents (solution candidates of the IGA). As a property of the parallel distributed IGA, in some generations, solution candidates are exchanged between the users: the best solution candidates in the same generation are exchanged (Fig. 1). With the exchange, each of the users is affected by other users' feelings. It is expected that good solution for all of the users is obtained.

Based on the concept of the proposed IGA, we constructed an IGA system for fundamentally investigating efficiencies of the proposed IGA. Aim of the IGA system is to create a short music melody commonly affording certain image to multiple users. The music melody is composed of sixteen successive music notes having equal length and velocity, and key of the notes was treated as gene of IGA individual. Listening experiment with the IGA system was conducted for investigating fundamental efficiencies of the proposed IGA. Note that this paper is an extend version of our study [19], and detail extension are as follows; parameter set of the IGA system was adjusted; listening experiment was conducted again with a larger number of subjects; frequency of the exchange of GA individual was increased; evaluating experiment was added.

In the next section, related studies including basic scheme of IGA are explained. The proposed IGA for creating music melody and its system are described in Section 3. Experiment for investigating fundamental efficiency of the proposed IGA and its results are presented in Section 4 and 5, respectively. The effectiveness of the proposed IGA is discussed in Section 5. Finally, we conclude the present study in Section 6.

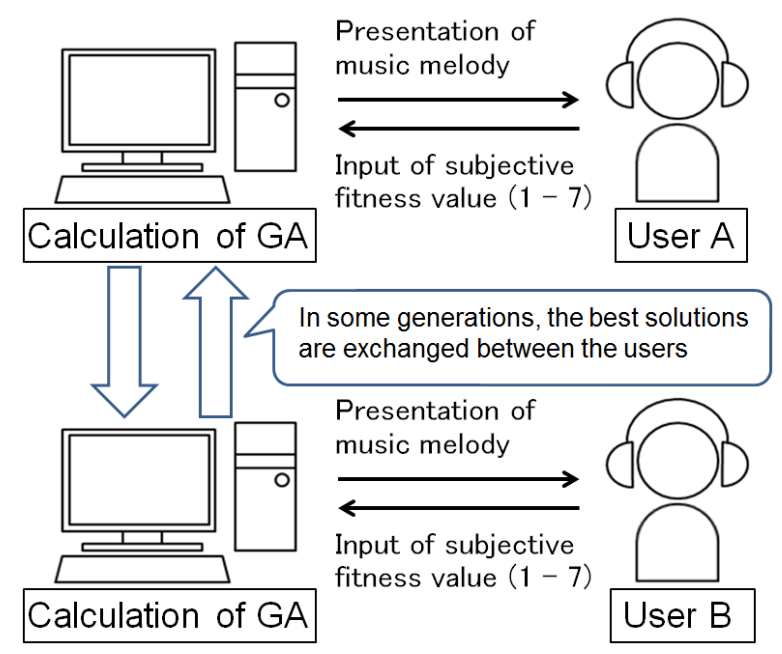

Figure 1: A scheme of the proposed Interactive Genetic Algorithm for creating music melodies suited to multiple users 


\section{Related Works}

\subsection{Genetic Algorithm and Interactive Genetic Algorithm}

GA is one of the most famous evolutionary algorithms and is widely used in various areas as optimizing method [17][18]. GA imitates evolution process of creatures for finding optimal solution in a certain problem. In the process of GA, individuals are corresponded to solution candidates, and gene of individuals plays a role of cord of solution candidate. General IGA expands GA's search ability by obtaining user's subjective evaluation as evaluation value for each of solution candidates.

Fig. 2 shows a flow chart of typical GA and IGA. Orange broken line suggests an area where human user evaluates GA individuals in IGA. Details of each process of GA and IGA are as follows:

(1) Initialization: GA and IGA begin its search with a creation of an initial population. In most of cases, values of individuals in the initial population are defined with random number in a range of upper and lower limits.

(2) Presentation: In GA, this process is considered that individuals are set in a function to obtain their fitness value. In IGA, individuals are converted to certain contents and are presented to the user. Method of the presentation is due to type of problem and individuals. In a case that individuals are pictures or graphics, several individuals would be presented in a same time so that the user can evaluate them comparatively. However, with time-sequential media contents such as music, sound and fragrance, individuals are presented sequentially because the user cannot evaluate multiple individuals in a same time.

(3) Evaluation: In GA, the function returns fitness value of individual. In IGA, the human user subjectively evaluates the presented individual by scoring or selection.

(4) Selection: To create the population in the next generation, parents of them are selected from the current generation. The selection is performed based on fitness value of individuals: individuals having higher fitness value get higher chance to be parent.

(5) Crossover: Crossover is one of the GA operations. In a process of creating an offspring (individual in the next generation), gene of offspring is defined by crossover of parents' genes.

(6) Mutation: Mutation is another GA operation. It changes parts of value of the gene.

The processes (2) to (6) are repeated. Terminate check plays a role to finish GA and IGA processes when pre-defined number of generations is finished. The IGA processes are also finished when the user is satisfied with the created individuals.

\subsection{Interactive Genetic Algorithm for Multiple Users}

Although IEC is originally used for creating media contents suited to each user, some previous studies proposed IEC for many users with various schemes. In situations with many people, using media contents suited to all of them is important. The previous IECs are as follows. All of them employed GA as evolutionary algorithm and had a purpose to optimize visual contents.

Akase et al. proposed an IGA that summarizes multiple users' subjective fitness values into one fitness value by calculation [11]. A target of their work was 3D room layout. Takenouchi and his colleagues proposed IECs with vote by multiple users [12][13][14]. In their IECs, each user votes to a better one from pair of individuals. The individuals gathered larger number of votes has chance to retain and make their offspring. Targets of their works were optimizing design of 
computer graphics of shoe, signboard, and clothes.

Miki and his colleagues proposed an island type IGA which is composed of some independent groups, and evolutionary process is performed in each group [15][16]. They named the IGA as a parallel distributed IGA. In their method, each of the users evaluates GA individuals in each group, and sometimes GA individuals are exchanged between each other group. In their first trial [15], synchronization of timing of the exchange was needed. It may become a problem if many users participate through network. To solve the problem, they proposed an asynchronous IGA method that keeps good individuals in a storage device [16].

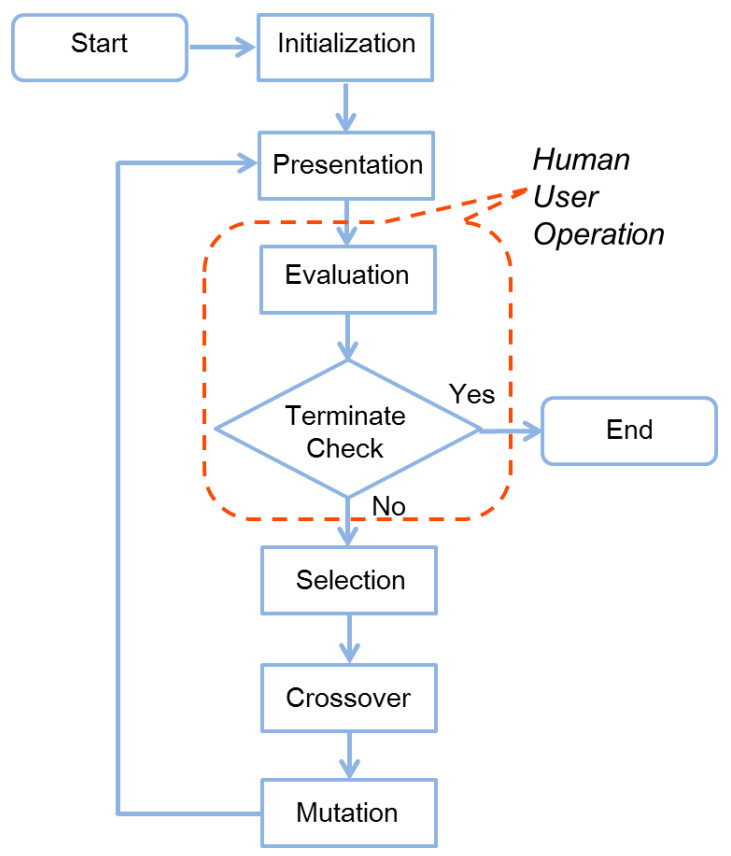

Figure 2: A flow chart of general Genetic Algorithm and Interactive Genetic Algorithm. Orange broken line shows processes that user evaluates individuals in Interactive Genetic Algorithm

\section{Interactive Genetic Algorithm for Creating Music Melody}

\subsection{IGA for Creating Music Melody Suited to Multiple Users and Construction of Its System}

Referring to the parallel distributed IGA [15], the present study proposed a distributed IGA creating music melody suited to multiple users. In situations such as waiting room of a hospital, a store, and a home party, music pieces play important roles creating atmosphere. From this perspective, obtaining music piece affording a certain common feeling to all listeners is demanded for creating atmosphere.

Outline of the proposed IGA is shown in Fig. 1. Each of the users performs IGA processes by evaluating music melodies presented from the IGA system with scoring or selection. In the end of the same generation, the best melodies are exchanged each other. In the exchange, the best individual of the user A is send to the IGA of the user B. In the IGA of the user B, an individual except the best individual is overwritten by the best individual of the user A, vice versa. If there are more two users, each of the users obtains the best individual of one of other participants. 


\subsection{Construction of A System based on the Proposed IGA}

As an implementation for investigating the fundamental efficiencies of the proposed IGA, a system based on the proposed IGA is constructed. As shown in Fig. 1, the IGA system is for only two users. Each of the two users individually evaluates presented music melodies repeatedly.

By using Musical Instrument Digital Interface (MIDI) format, GA individuals are converted to music file. Fig. 3 (a) illustrates correspondence of values in GA individual and key. With MIDI format, correspondence of value and music key is defined by referring to previous IEC studies [20][21][22]. In the IGA system, music keys in two octaves from 60 to 84 are used. The music melody created by the IGA system was composed of sixteen successive music notes having equal length and velocity. Key of the notes was treated as gene of IGA individual. Fig. 3 (b) shows an example of music melody and attached music chord progression. Music chord progression called Canon chord is attached to the melody when it is presented to the subjects. Fig. 3 (b) shows original MIDI file that is used for creating variety of music melodies by changing its key of music notes. In this case, all of sixteen genes in GA individual are set as 60 .

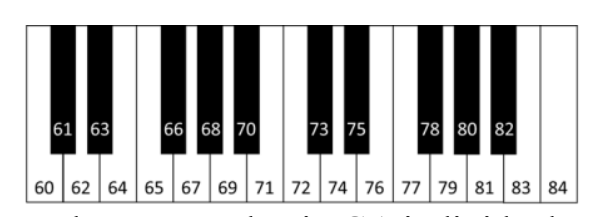

(a) Correspondence between value in GA individual and music key

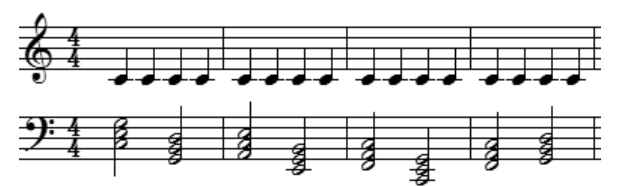

(b) Original MIDI file used in the IGA system

Figure 3: Part of construction of the IGA system showing relationship between values used in the IGA system and music melody

\section{$4 \quad$ Listening Experiment}

To investigate the fundamental efficiency of the proposed distributed IGA for music composition, listening experiment was conducted with the IGA system described in the previous section. Target of creation was "bright" music melody which affords common impression between subjects and is suited to a music chord progression. Sixteen subjects participated in the experiment.

\subsection{Searching Experiment}

The subjects were divided into eight pairs: two subjects participated in the evaluation process simultaneously. Through twenty-one generations, the subjects evaluated eight GA individuals in each generation: total evaluation time was 168 . The subjects individually listened the presented music melodies via a headphone and evaluated them by scoring from 1 (extremely dark impression) to 7 (extremely bright impression) with Semantic Differential (SD) method [23]. The experiment started from the 0th generation. In the end of the $3 \mathrm{rd}, 7 \mathrm{th}, 11 \mathrm{th}, 15 \mathrm{th}$, and 19th generations, the exchange of the best solutions between the two subjects was performed. Note that the exchange was a kind of synchronized event.

Table 1 summaries GA parameters used in the experiment. In the IGA system, roulette shuffle 
selection and elitism strategy were employed, and 1-point cross over and mutation were occurred in gene of with $95 \%$ and $5 \%$ respectively. In a case mutation occurred, the value of gene was changed from -4 to 4 excepting 0 . After finishing evaluation tasks, a questionnaire asked the subjects impressions of the presented music melodies and the evaluation task. The questionnaire is composed of five items shown in Table 2. The subjects responded to these five items with SD method in 5 point scale: 5 meant strongly agree, and 1 point meant strongly disagree.

Table 1: Parameter Set of IGA

\begin{tabular}{|l||l|}
\hline Number of Generation & 21 \\
\hline Number of Individuals & 8 \\
\hline Selection & $\begin{array}{l}\text { Roulette Shuffle and Elit- } \\
\text { ism Strategy }\end{array}$ \\
\hline Crossover & One-point Crossover (95\%) \\
\hline Mutation & Uniform Mutation (5\%) \\
\hline
\end{tabular}

Table 2: Questionnaire Items

\begin{tabular}{|l|l|}
\hline & Item \\
\hline \hline Q1 & Are you satisfied with the obtained melodies? \\
\hline Q2 & Are you tired with the experimental task? \\
\hline Q3 & Were there any unexpected music melodies? \\
\hline Q4 & Can you compose music melodies efficiently? \\
\hline Q5 & Does this system help your imagination? \\
\hline
\end{tabular}

\subsection{Evaluating Experiment}

Fitness values obtained in the searching experiment are considered as relative values in a same generation. Thus, to investigate the efficiency of the proposed IGA precisely, comparison of GA individuals obtained in the final and the initial generations is needed. After at least one hour from finishing the searching experiment, the two best individuals created in his/her own searching experiment were afforded to the subject. Order of the two best individuals was randomized and counter-balanced. With the same experimental set and the same evaluation scale in the searching experiment were used in the evaluating experiment.

\section{Experimental Results}

As an experimental result, Fig. 4 shows progress of mean and maximum fitness values between all of the sixteen subjects: these values were obtained after calculating each subject's mean and maximum fitness values in each generation. Green underline denotes generations where the subjects listened to and evaluated the exchanged GA individuals: in the end of the previous generation of these generations, the best individual of one subject was copied to another subject.

Throughout twenty-one generations, slight increase in both of mean and maximum fitness values was observed. In the generations where the subjects evaluated the exchanged GA individual, no-special increases in the fitness value were observed: only in the 8th generation, a little rapid increase in the fitness values was observed.

Fig. 5 shows progress of mean fitness values in two pairs of the subjects. In the left graph, mean fitness values of two subjects A and B were almost same level throughout twenty-one generations excepting from the 4 th to the 8 th generations where a small difference was observed. Both of them started 2 point in the initial generation and reached over 4 point in the final generation. Obvious changes in the fitness value were not observed in the generations where the subjects evaluated the exchanged GA individual excepting the 16th generation where almost 1 point increases from the 15th generation were observed in the fitness value in both of the subjects: 1 point increase is relatively large increase.

While the almost same trend was observed in Subject A and B, right graph of Fig. 5 shows a 
quite different progress in the fitness value between the subjects. Mean fitness value in Subject C changed slightly between the generations. On the other hand, the mean fitness value in Subject D widely changed with a repetitive up and down. In the progress of the mean fitness value in Subject $\mathrm{D}$, some peaks were observed during its change, and these peaks were observed in the 4th, 12th, and 20th generations where the exchanged GA individual was evaluated.

Fig. 6 shows mean values and standard deviation for the five questionnaire items. In the all items, relatively high value over 3 point were observed. Especially, in Q5 related to imagination of new music melody, relatively higher value near to 4 point was observed.

Fig. 7 shows fitness values obtained in the evaluating experiment where the subject evaluated the representative best individuals picked up from the initial and the final generation. By comparing the fitness values, increase in the mean fitness value was observed. However, with a statistical test, no significant increase was observed.

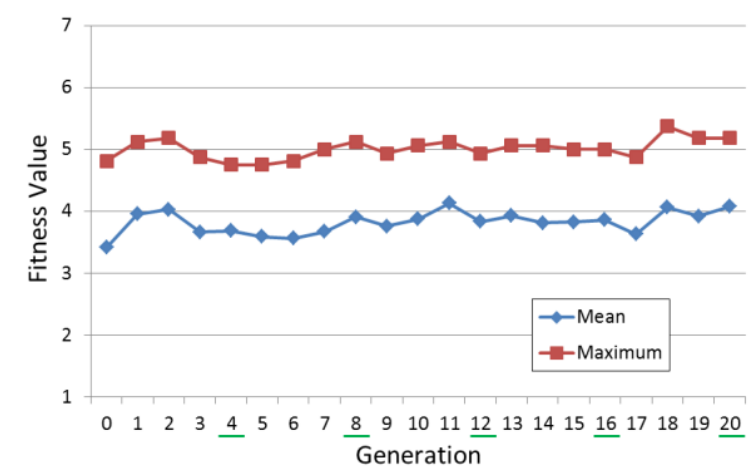

Figure 4: Progress of mean and maximum fitness values between all of sixteen subjects
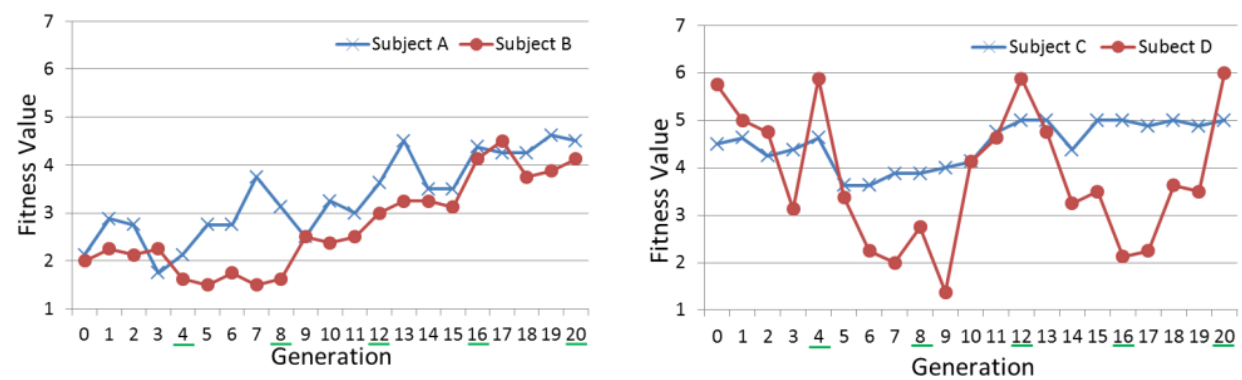

Figure 5: Examples of progress of mean fitness value in two pairs: (left) Subject A and B, (right) Subject C and D

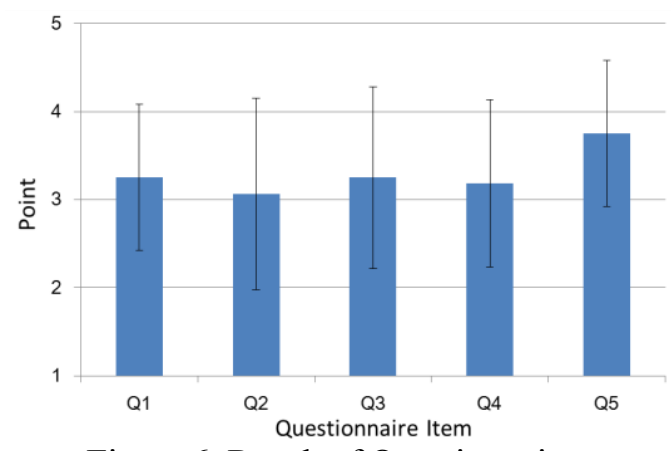

Figure 6: Result of Questionnaire

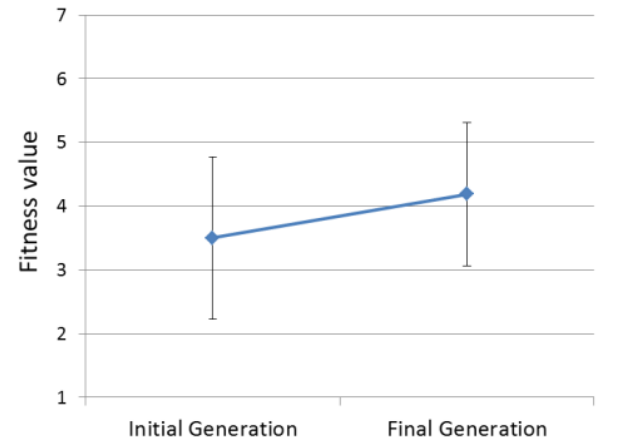

Figure 7: Result of Evaluating Experiment 


\section{Discussion}

In the searching experiment, the progress of mean and maximum fitness values between all of the subjects showed a slight increase throughout twenty-one generations. Result of the evaluating experiment, a weak trend of increase in the mean fitness value was observed. These increases were not significant, however, the weak trend of increase means that the proposed IGA has a possibility to find better music melodies.

A little rapid increase in the mean fitness value between all of the subjects was observed in the 8th generation where the subjects evaluated the exchanged GA individual. By observing samples of two pairs of subjects, resemble trends were observed in some generations. The relationship between the increase in the fitness value and the exchange is important key in the proposed IGA. The exchange might dedicate to the increase in the fitness value in terms of improving searching process in each of the subjects. To clarify the efficiency of the exchange in the proposed IGA on the increase in the fitness, further study with comparing experiment including conditions with/without the exchange is needed. Moreover, we need to investigate the fitness value of the exchanged GA individuals.

In the constructed IGA system, the exchange of the best GA individual was performed in the same time. Therefore, one of the two subjects had to wait to finishing another subject's evaluation. The waiting might give the subject a kind of bias for the evaluation in the next generation. This problem will be solved by referring to the asynchronous parallel distributed IGA [16] using a storage device. After solving the problem of the synchronization, we plan to have the same type of listening experiment.

In the listening experiment, timing of the exchange was set in the 3rd, 7th, 11th, 15th, and 19th generations. Appropriate timing of the exchange is also a key factor of the parallel distributed IGA, because too much frequent exchange might interrupt the efficient search. Employing adaptive method related to state of search might be effective to solve this problem.

\section{Conclusion}

The present study proposed the distributed IGA for creating music melodies suited to multiple users' feeling. The proposed IGA is based on the parallel distributed IGA [15] that exchanges the best GA individuals between the users. The system based on the proposed IGA was constructed for investigating the fundamental efficiency of the proposed IGA. The experimental results showed a possibility of the proposed IGA to create music melodies suited to the multiple users by means of the trend of increase in the mean fitness value. Additionally, with the questionnaire items, most of the subjects felt that the proposed IGA helps their imagination for creating new music melodies.

To investigate the efficiencies of the proposed IGA, further investigations with comparing experiment between with and without the exchange. Timing of the exchange must be key of improvement the proposed IGA, therefore, investigation of direct effect of the exchange is also needed by analyzing the fitness value of the exchanged GA individuals. Asynchronous function is not implemented on the IGA system in this study. We are planning to add the asynchronous function for enhancing the proposed IGA that gather multiple users' evaluation easily. 


\section{References}

[1] H. Takagi, "Interactive Evolutionary Computation: Fusion of the Capabilities of EC Optimization and Human Evaluation," Proc. the IEEE, vol.89, no.9, 2001, pp. 1275-1296.

[2] R. Dawkins, The Blind Watchmaker, Norton, 1986.

[3] M. Herdy, "Evolutionary optimization based on subjective selection -evolving blends of coffee," Proc. 5th European Congress on Intelligent Techniques and Soft Computing, 1997, pp. 640-644.

[4] M. Fukumoto and J. Imai, "Design of Scents Suited with User's Kansei using Interactive Evolutionary Computation," Proc. Kansei Engineering and Emotion Research 2010, 2010, pp. 1016-1022.

[5] M. Fukumoto, M. Inoue, and J. Imai, "User's Favorite Scent Design Using Paired Comparison-based Interactive Differential Evolution,” Proc. IEEE CEC2010, 2010, pp. 4519-4524.

[6] M. Fukumoto, K. Kawai, M. Inoue, and J. Imai, "Interactive Tabu Search with Paired Comparison for Optimizing Fragrance,” Proc. IEEE SMC2013, 2013, pp. 1690-1695.

[7] M. Fukumoto, S. Koga, M. Inoue, and J. Imai, "Interactive Differential Evolution Using Time Information Required for User's Selection: In A Case of Optimizing Fragrance Composition,” Proc. IEEE CEC2015, 2015, pp. 2192-2198.

[8] H. Nishino, K. Takekata, M. Sakamoto, B. A. Salzman, T. Kagawa, and K. Utsumiya, "An IEC-Based Haptic Rendering Optimizer,” Proc. IEEE WSTST’05, 2005, pp. 653-662.

[9] K. Takekata, B. A. Salzman, H. Nishino, T. Kagawa, and K. Utsumiya, “An Intuitive Optimazation Method of Haptic Rendering Using Interactive Evolutionary Computation," Proc. IEEE SMC2005, 2005, pp. 1896-1901.

[10] M. Fukumoto and T. Ienaga, "A Proposal for Optimization Method of Vibration Pattern of Mobile Device with Interactive Genetic Algorithm," Proc. HCII2013, vol.11, 2013, pp. 264-269.

[11] R. Akase and Y. Okada, "Web-based Multiuser 3D Room Layout System Using Interactive Evolutionary Computation with Conjoint Analysis," Proc. of the 7th International Symposium on Visual Information Communication and Interaction, 2014, pp. 178-187.

[12] H. Takenouchi, M. Tokumaru, and N. Muranaka, "An interactive genetic algorithm with tournament evaluation of individuals by multiple people," Proc. SCIS\&ISIS2008, 2008, pp. 1061-1066.

[13] H. Takenouchi, H. Inoue, and M. Tokumaru, "Signboard design system through social voting technique," Proc. ISIC2014, 2014, pp. 14-19.

[14] M. Sakai, H. Takenouchi, and M. Tokumaru, "Design support system with votes from multiple people using digital signage,” Proc. ISIC2014, 2014, pp. 26-31. 
[15] Y. Ogawa, M. Miki, T. Hiroyasu, and Y. Nagaya, "A New Collaborative Design Method Based on Interactive Genetic Algorithms," eurogen, 2001.

[16] M. Miki, Y. Yamamoto, S. Wake, and T. Hiroyasu, "Global Asynchronous Distributed Interactive Genetic Algorithm,” Proc. IEEE SMC2006, vol.4, 2006, pp. 3481-3485.

[17] J. H. Holland, Adaptation in Natural and Artificial Systems: An Introductory Analysis with Applications to Biology, Control and Artificial Intelligence. The University of Michigan Press, USA, 1975.

[18] D. Goldberg, Genetic Algorithms in Search, Optimization and Machine Learning. Addison-Wesley Professional, USA, 1989.

[19] M. Fukumoto and T. Hatanaka, "Parallel Distributed Interactive Genetic Algorithm for Composing Music Melody Suited to Multiple Users' Feelings," Proc. ICIS2016, 2016, pp. 831-836.

[20] M. Miki, H. Orita, S. H. Wake, and T. Hiroyasu, "Design of Sign Sounds using an Interactive Genetic Algorithm," Proc. the IEEE SMC2006, vol.4, 2006, pp. 3486-3490.

[21] M. Fukumoto, T. Hazama, and J. Imai, "Evolutionary Computation for Creating Musical Melody based on User's Physiological Index,” Proc. SCIS\&ISIS2008, 2008, pp. 247-252.

[22] M. Fukumoto, "An Efficiency of Interactive Differential Evolution for Optimization of Warning Sound with Reflecting Individual Preference," IEEJ Transactions on Electrical and Electronic Engineering, vol.10, issue S1, 2015, pp. S77-S82.

[23] C. E. Osgood, G. J. Suci, and P. Tannenbaum, The measurement of meaning, University of Illinois Press, 1957 